[8] Ghandhi, S. K., Theory and Practice of Microelectronics. New York: Wiley, 1968.

[9] Sze, S. M., Physics of Semiconductor Devices. New York: Wiley-Interscience, 1968, pp. 90, 92, 113, 214.

[10] Brown, W. D., Semiconductor Device Degradation by High
Amplitude Current Pulses, Sandia Laboratories Document SCDC-72-2288, July 1972.

[11] Alexander, D. R., R. J. Karaskiewicz, and P. A. Young, Final Report for Electronics Component Modeling and Testing Program, AFWL-TR-78-62, 1978.

\title{
Radiation Effects on Silicon Charge-Coupled Devices
}

\author{
JOSEPH M. KILLIANY, MEMBER, IEEE
}

\begin{abstract}
The permanent ionizing and neutron radiation-induced degradation in silicon charge-coupled devices, (CCD's), along with transient upset effects, are reviewed. The operation of a threshold voltage insensitive $\mathrm{CCD}$ input technique in a total dose radiation environment is evaluated. CCD structural design rules for decreasing ionizing radiation sensitivity are presented. The increased total ionizing dose tolerance of CCD's fabricated with a radiation hard oxide is described. Liquid nitrogen temperature irradiation effects in CCD's are discussed.
\end{abstract}

\section{INTRODUCTION}

C HARGE-COUPLED devices (CCD's) have potentially extensive applications in optical imaging, signal processing, and serial memories. They have small size, low-power consumption, and high reliability. Such characteristics make CCD's attractive for certain space and military missions provided the devices can satisfy the radiation hardness requirements.

The radiation-produced damage in a semiconductor device depends on its relative sensitivity to ionization and bulk displacement effects. Surface-controlled devices (MOS) tend to be limited by the ionization produced, since ionization leads to charge buildup in insulator layers and to an increase in surface state density at the insulator-semiconductor interface. The characteristics of bulk-effect devices are usually degraded by displacement damage, since this damage can significantly decrease carrier concentration, carrier mobility, and carrier lifetime [1]. CCD's are sensitive to both surface and bulk radiation damage effects. They are also very susceptible to transient radiation induced loss of stored information.

This paper has been organized in the following manner. First, the basic operating principles of CCD's are presented. Second, the basic radiation induced degradation mechanisms in silicon devices are reviewed. Then the surface, bulk and transient radiation effects in CCD's are discussed. Radiation hardening techniques are presented in the fourth section, and liquid nitrogen temperature radiation effects are described in the fifth section. Finally, the current status of understanding of radiation effects on CCD's is summarized.

Manuscript received April 15, 1978; revised August 7, 1978.

The author is with the Naval Research Laboratory, Washington, DC, 20375.

\section{CCD OPERATION}

The details of CCD operation have been discussed in a number of papers and books [2]-[5]. Those CCD concepts which are important to the treatment of radiation effects are briefly reviewed here for convenience.

A charge-coupled device consists of an array of closely spaced MOS capacitors which can collect and store minority carrier charge packets in localized potential wells. The charge is transferred from one potential well to the adjacent one by manipulating the voltages applied to the capacitor electrodes. However, the transfer of charge from one CCD potential well to another is not a lossless process. Charge may be trapped in interface states or bulk states. Potential well irregularities may prevent some of the charge from transferring. Improper clocking (wrong phasing or too fast) may limit the amount of charge which is transferred. The fraction of the charge which is successfully transferred from one well to the next is called the transfer efficiency $\eta$. Since the transfer efficiency in a good device is approximately unity, it is often more convenient to deal with the transfer inefficiency $\epsilon$, which is defined by $\epsilon=1-\eta$.

In the first CCD and in many current designs, the signal charge is stored and transported in a region at the semiconductor surface. This type of device is called a surface channel CCD. The transfer efficiency in a surface channel CCD is limited severely by the effects of interface-state trapping unless a constant background or bias charge (fat zero) is maintained in the potential wells to keep the interface states filled so that trapping of the signal charge is minimized. The transfer inefficiency in a stale-of-the-art surface-channel device is approximately $1 \times 10^{-4}$ when measured with a bias charge equal to 20 percent of the signal handling capacity.

In order to avoid these interface-state effects, a buried channel CCD approach is used. A $p-n$ junction is introduced into the semiconductor a short distance below the insulatorsemiconductor interface, and the resulting potential profile keeps the signal charge confined in the semiconductor at some distance from the surface. The signal charge packet does not interact with the interface states, and therefore a bias charge is not required for efficient transfer. However, a small fraction of the signal charge is trapped by bulk states in the silicon leading to a charge transfer inefficiency in the $10^{-5}$ range. 
For most applications, the total fraction of the charge which is lost in transfer from input to output is of primary importance. This fraction is, of course, given by the $N \epsilon$ product, where $N$ is the number of transfers involved in going from input to output. Generally an $N \epsilon$ product of 0.1 or less is desired for linear applications. Digital applications usually are less restrictive.

Even in the absence of input signal, the CCD potential wells are continuously being filled by thermally generated charge. The resulting output is known as the dark current. Three sources of dark current are interface states at the $\mathrm{Si}-\mathrm{SiO}_{2}$ boundry, bulk traps in the depletion region of the semiconductor, and bulk traps in the neutral semiconductor within a diffusion length of the surface [4]. The dark current determines the amount of time that a potential well can exist in the device. The dark current is a strong function of temperature, decreasing by a factor of 2 in silicon for every $8^{\circ} \mathrm{C}$ decrease in temperature [6]. The thermal carrier generation rate is usually expressed in terms of dark current density $J_{D}$. Dark current densities in the $1-10 \mathrm{nA} \mathrm{cm}^{-2}$ range are observed in state-ofthe-art devices.

An additional parameter of interest is the maximum amount of signal charge that can be stored in the CCD potential well. This quantity is called the full-well capacity and is generally given as the number of electrons (or holes) that can be stored in a well without overflowing into adjacent wells. Typical capacity of a CCD potential well having a gate area of $1 \times 10^{-6} \mathrm{~cm}^{2}$ would be $2 \times 10^{6}$ carriers in a surface-channel structure and $6 \times 10^{5}$ carriers in a buried channel device.

\section{RADIATION EFFECTS IN SEMICONDUCTOR DEVICES}

The basic effects of radiation on semiconductor devices have been described by Gregory [1]. The salient features of that revicw will be briefly outlined here as a background for the discussion of expected radiation effects in CCD's.

High-energy radiation deposits energy in semiconductor materials via two mechanisms, atomic collisions and electronic ionization. The relative importance of these two mechanisms depends both on the type of radiation and the nature of the device. Electrons, protons, and gamma rays deposit most of their energy via the ionization process, while fast neutrons deposit up to 50 percent of their energy in atomic displacement damage. MOS devices are more sensitive to ionizing radiation, while the characteristics of bulk-effect devices such as bipolar transistors, are usually degraded by displacement damage.

Numerous studies have shown that ionizing radiation causes failure of MOSFET devices due to two mechanisms: 1) trapped charge buildup in the silicon dioxide layer and 2) an increase in the density of trapping states at the silcon-silicon dioxide interface. Ionizing radiation generates electron-hole pairs in the silicon dioxide. The electrons are swept out of the oxide, but some of the holes are trapped permanently producing a negative threshold voltage shift. The size of the threshold voltage shift varies with the magnitude and polarity of the applied gate bias during irradiation. Positive gate-substrate bias results in a greater threshold voltage shift since the holes are trapped near the silicon surface where they will exert maximum influence on the semiconductor. An increase in interface state density causes a decrease in MOSFET transconductance.

In addition to these permanent degradation mechanisms, ionizing radiation produces electron-hole pairs in the silicon substrate during irradiation. Carriers produced in device depletion regions, or within a diffusion length of these regions, can produce photocurrent at the device terminals. These photocurrents can cause large transients in linear circuits and can cause error in the information stored by logic circuits.

Fast neutron irradiation produces displacement damage in the silicon, which leads to significant decreases in carrier concentration, carrier mobility, and minority carrier lifetime. Decrease in carrier concentration and minority carrier lifetime reduction are the dominant failure mechanisms in most neutron irradiated bulk semiconductor devices. Mobility degradation does not become severe in silicon until neutron fluence exceeds $10^{15}$ neutrons $/ \mathrm{cm}^{2}$.

\section{SURFACE DAMAGE EFFECTS}

Since CCD's are MOS devices, ionizing radiation causes a buildup of positive charge in the gate oxide and an increase in trapping states at the silicon-silicon dioxide interface. The resulting negative flatband voltage shift causes a change in the CCD operating bias, while the interface state density increase reduces the charge transfer efficiency in surface channel devices and increases the dark current density in both buried and surface-channel structures. Consequently, nonhardened devices are unsuitable for most room-temperature applications requiring a dose tolerance greater than $5 \times 10^{4} \mathrm{rad}(\mathrm{Si})$. The details of these radiation-induced failure mechanisms are presented in the following paragraphs.

\section{Positive Charge Buildup in the Oxide}

For a given oxide technology, the smallest flatband voltage shifts are expected for $n$-buried channel and $p$-surface channel devices because the gate voltage is negative with respect to the channel potential. The effects of positive space charge buildup in the oxide on CCD operation will be demonstrated using a simple shift register of the type shown in Fig. 1. The CCD shift register is separated into the following three parts for the purpose of discussing radiation effects:

1) input structure, consisting of the input diode, input gates, and first clock gate;

2) charge transfer section, consisting of the clocked electrodes;

3) output detector-amplifier, consisting of the output gate, reset gates, output diode, reset MOSFET, and source follower MOSFET.

1) Input Structure: The input structure of the shift register is the most radiation sensitive section of the device unless a threshold insensitive input is employed. Input techniques, such as dynamic injection, operate by leaking charge into the CCD potential well over the barrier formed by the input transfer gates, $\phi_{\text {IN BM }}$ and $\phi_{\text {INSM }}$. The quantity of charge injected is 


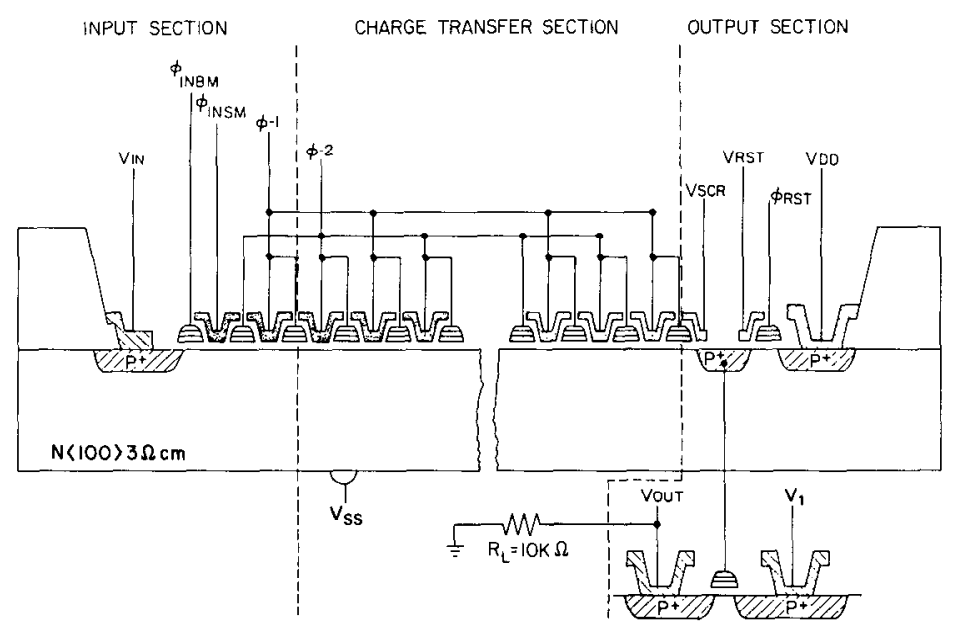

Fig. 1. Cross section of two-phase $p$ surface channel CCD shift register. Device is shown separated into three sections: input, charge transfer section, and output.

given by [7]

$$
Q_{s}=\frac{\beta}{2}\left(V_{\mathrm{GS}}-V_{T}\right)^{2} T
$$

where $V_{\mathrm{GS}}$ is the gate to source voltage and $V_{T}$ is the input gate threshold voltage. $\beta$ is a constant dependent on the device geometry, and $T$ is the time available for filling the potential well. An input of this type is extremely sensitive to small changes in gate to source bias and typically tolerates only $10^{3}$ $\mathrm{rad}(\mathrm{Si})$ before requiring adjustment. An example of the shift in the CCD transfer curve for the dynamic injection input is shown in Fig. 2 [8]. The negative flatband voltage shift will eventually cause input saturation for $n$-surface and $n$-buried channel devices and input cutoff for $p$-surface and $p$-buried channel structures.

Threshold voltage shifts up to -5 volts have been accommodated by the input structure when the potential equilibration input, a threshold insensitive technique, was employed [7], [8]. The manner in which the potential equilibration input technique operates is illustrated in Fig. 3. A potential well is established under the phase 1 and 2 electrodes $(P 1$ and $P 2)$. The input diode (ID) is pulsed to cause charge to flow over the barrier established under the input gate (IG). The charge is prevented from blooming down the CCD channel by the barrier under the phase 3 gate $(P 3)$. While both transfer gates ( $P 1$ and $P 2)$ are still high, the diode voltage is returned to the resting level, and the excess charge is drained from under $P 1$ and $P 2$. The amount of charge retained in the CCD well is proportional to the difference in the channel potential under the $P 1-P 2$ e. lectrodes and the input gate (IG). Hence, to first order, the amount of signal charge injected in the CCD will be threshold insensitive for flatband voltage shifts of equal magnitude under the input and first transfer gates.

The CCD transfer curves generated while using the potential equilibration input technique are shown in Fig. 4. [8] The postirradiation transfers curves coincide with the preirradiation curve in the threshold insensitive region for threshold voltage shifts up to -3.6 volts. The maximum threshold voltage shift which could be accommodated in this particular de-

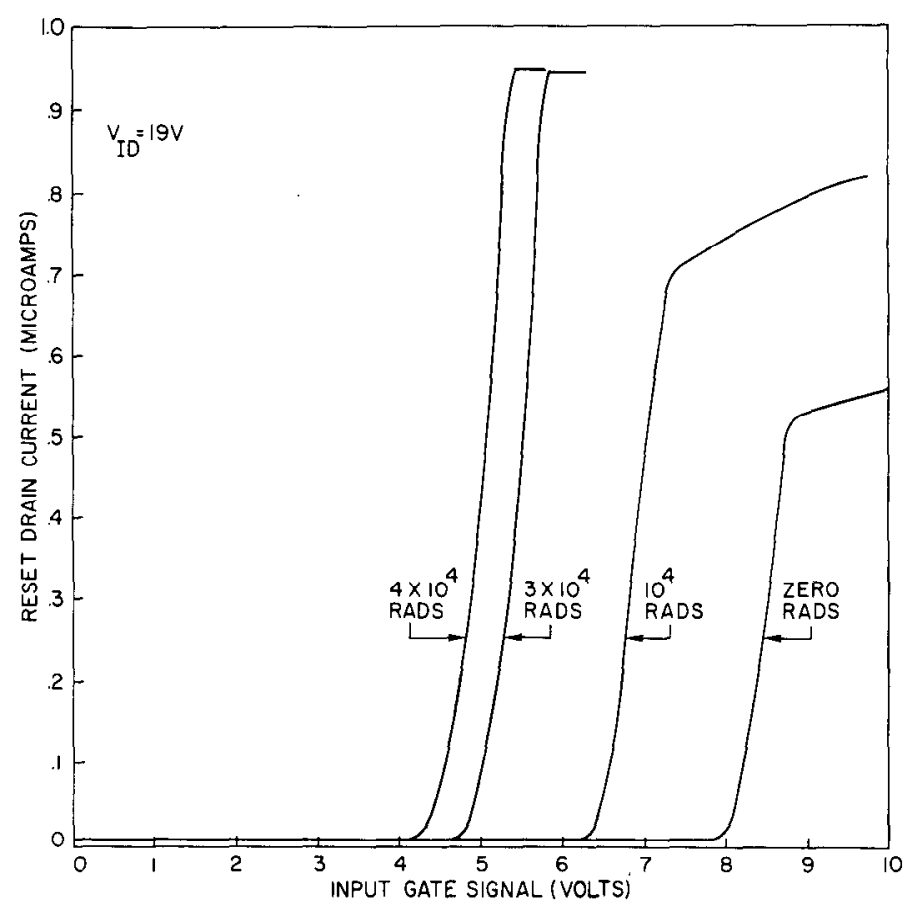

Fig. 2. CCD transfer curves for dynamic injection input as function of radiation dose.

vice while maintaining a reasonable signal injection capacity was -5.0 volts. The potential equilibration input has a limited flatband voltage shift accommodation because the channel potentials $\phi$ are a function of the flatband voltage, while the input diode voltage levels $V$ obviously are not [8]. In the case of the $n$-buried channel device shown in Fig. 3, a negative flatband voltage shift reduces the barrier under phase $3(P 3)$ which eventually allows charge to spill down the CCD channel. The magnitude of the threshold voltage shift accommodation could of course be increased by adjusting the input diode low level; this would, however, also reduce the signal handling capacity of the input. While the operation of the potential equilibration input was illustrated using a buried channel device, it should be noted that this technique is also applicable to surface channel structures. 


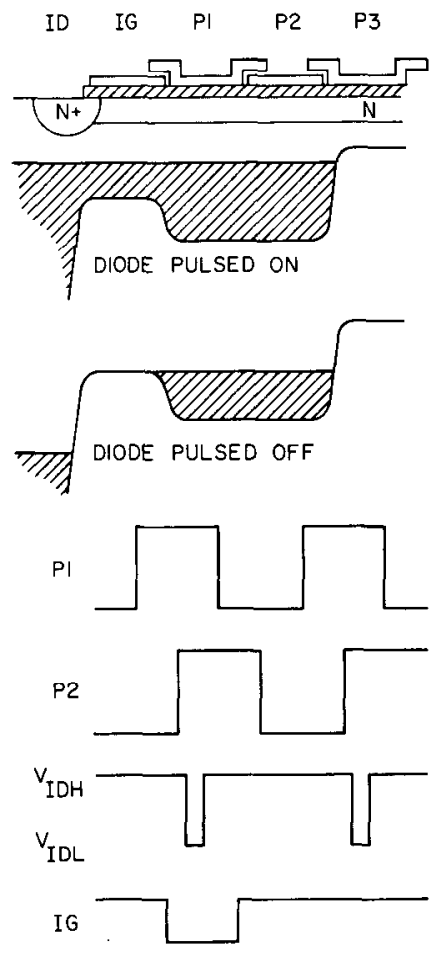

Fig. 3. Cross section of CCD input structure showing potential energy profile in silicon for potential equilibration input technique along with gate and diode voltagcs.

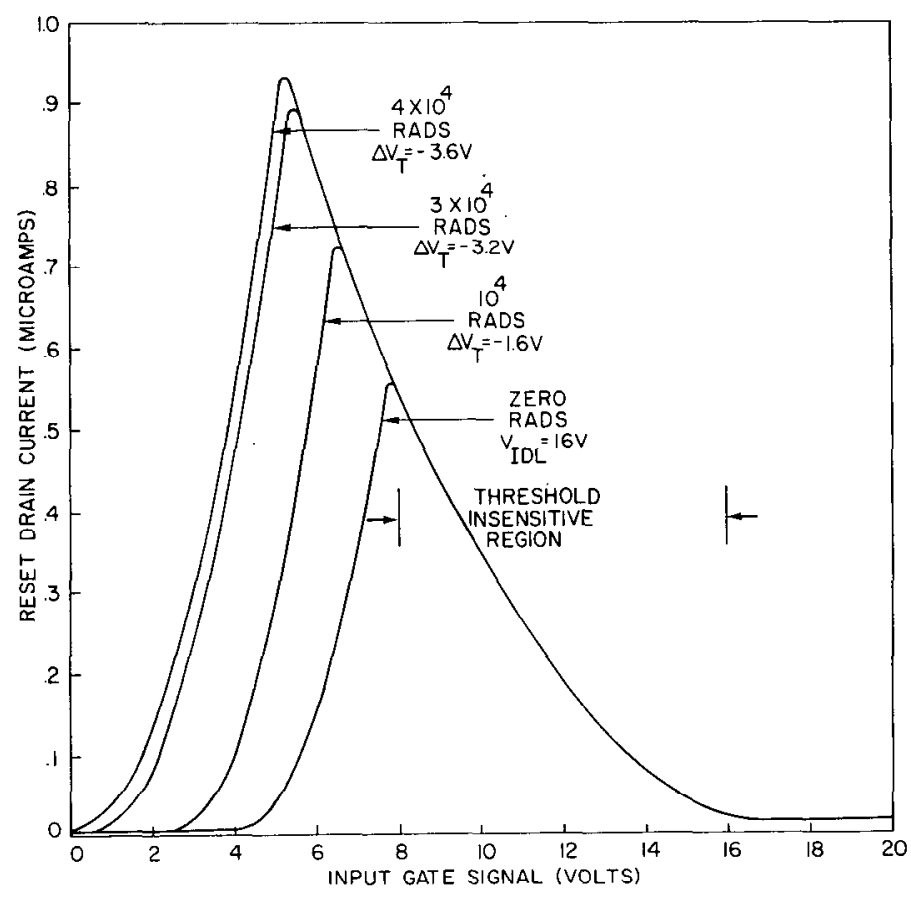

Fig. 4. CCD transfer curves for potential equilibration input as function of radiation dose.

Threshold tracking circuits, of course, may be employed to compensate for positive charge buildup in the oxide. However, the successful operation of these circuits on a CCD chip has not been reported.

2) Charge Transfer Section: The charge transfer process in a properly designed charge-coupled device is fairly insensitive to uniform transfer gate flatband voltage shifts. However, gate-to- gate nonuniformities may distort the potential profile in the CCD channel with increased trapping of the signal charge as a result. Unequal flatband voltage shifts on adjacent gates have also caused a severe loss in CCD signal handling capacity [9] . The difference in flatband voltage shifts between the aluminum and polysilicon gates in a stepped oxide structure is shown in Fig. 5. The unequal flatband voltage shifts reduced the signal handling capacity after $3 \times 10^{5}$ rad to 20 percent of the preirradiation value. A cross section of the two-phase stepped oxide shift register along with the pre- and postirradiation channel potential is shown in Fig. 6 .

A negative flatband voltage shift will cause the potential energy profile in the CCD channel to change in both surface and buried channel structures. Eventually, the channel will be driven out of depletion in $p$-surface and $n$-buried channel devices. The increase in the reset drain voltage $V_{\mathbf{D D}}$ required to operate an $n$-buried channel after irradiation is shown in Fig. 7 and is approximately equal to the flatband voltage shift [10] . A few volts of flatband voltage shift accommodation can be obtained in an $n$-buried channel structure simply by applying a reverse bias to the reset drain voltage $V_{\mathbf{D D}}$, several volts in excess of the preirradiation value required to deplete the channel. The flatband voltage shift tolerance of a $p$-surface channel device can be increased by either applying a positive substrate bias or a negative clock offset voltage.

3) Output Section: The CCD output is the least radiation sensitive part of the device. The input and charge transfer sections will usually. severely degrade before the output becomes inoperable. The reduced radiation sensitivity of the CCD output can be attributed to the following.

1) The reset MOSFET is used as a switch to reset the gate of the source follower transistor to the reference voltage $V_{\mathbf{D D}}$. Hence, the reset pulse levels can be set to values which will allow pre- and postirradiation operation.

2) The output MOSFET threshold voltage shift is smaller than the shift on the other CCD gates due to the reduced elec. tric field strength in that oxide. Typical electric field values in the transfer gate and source follower gate oxides are $0.9 \times 10^{6} \mathrm{~V} / \mathrm{cm}$ and $0.25 \times 10^{6} \mathrm{~V} / \mathrm{cm}$, respectively.

3) The source follower output is ac coupled. Hence, small shifts in the dc operating point of the output MOSFET are of little consequence.

4) Since the output transistor is operated as a source follower, the output gain, $A_{V}$, will be relatively insensitive to changes in the transconductance $g_{m}$ for values of $g_{m} R_{L} \gg 1$.

$$
A_{V} \cong \frac{g_{m} R_{L}}{1+g_{m} R_{L}}
$$

where $R_{L}$ is the source follower load resistor.

\section{Interface-State Density Increase}

The irradiation produced increase in trapping state density at the silicon-silicon dioxide interface reduces the charge transfer efficiency in surface channel devices and increases the surface component of the dark current density in both surface and buried channel CCD structures. The transfer inefficiency $\epsilon_{\boldsymbol{s}}$ attributable to interface-state trapping is directly propor- 


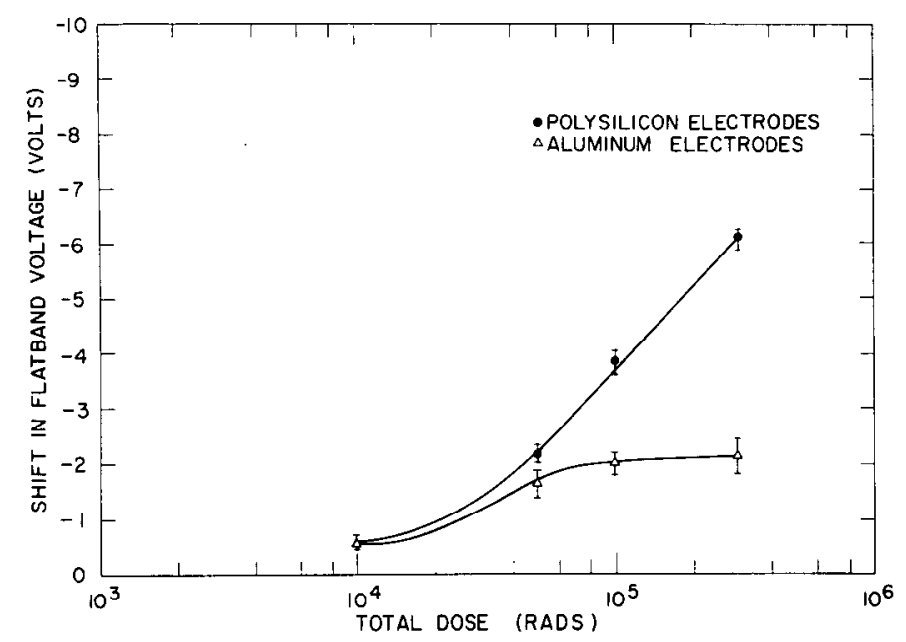

Fig. 5. Unequal flatband voltage shifts for polysilicon and aluminum gate electrodes as function of dose.

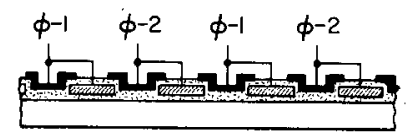

(a)

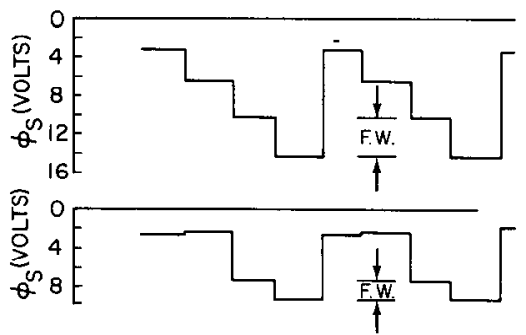

Fig. 6. Distortion of channel potential profile in two-phase surface channel CCD caused by unequal flatband voltage shifts for adjacent gate elecrodes.

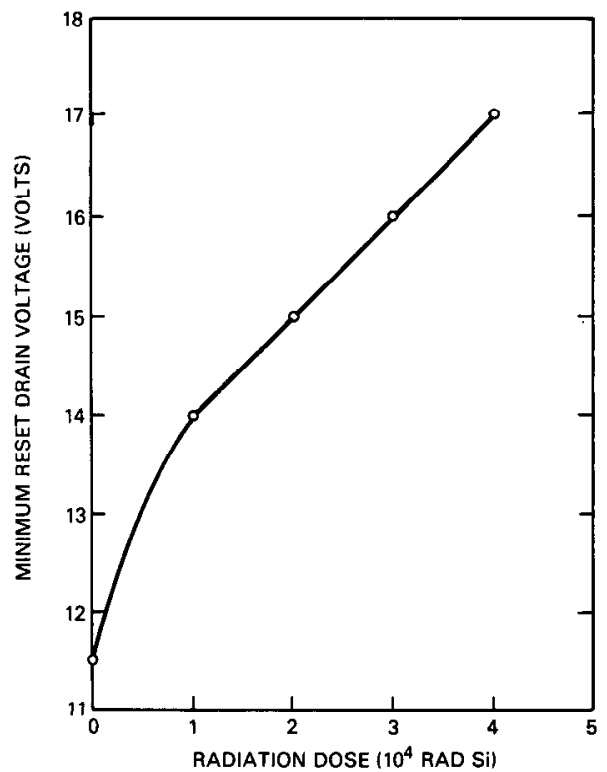

Fig. 7. Minimum reset voltage $V_{\mathrm{DD}}$ required to deplete channel in $n$-buried channel CCD as function of dose. tional to the interface-state density. An empirical expression for the transfer inefficiency is given by [11]

$$
\epsilon_{s}=\frac{1}{n_{s}+n_{s, 0}} k \mathrm{TN}_{s s} \ln \left(1+\frac{2 f c}{k_{1} n_{s, 0}}\right)
$$

where

$n_{s, 0}$ number of bias charge carriers per unit area,

$n_{s}$ number of signal carriers per unit area,

$N_{s s}$ interface state density in $\mathrm{cm}^{-2}(\mathrm{eV})^{-1}$,

$f_{c}$ clock frequency.

$k_{1}$ a constant parameter that depends on the trapping cross section $\left(k_{1} \cong 10^{-2} \mathrm{~cm}^{2} / \mathrm{s}\right)$.

Most of the interface-state trapping in surface channel devices operated with an adequate bias charge takes place at the edges of the gate electrodes. Interface states in those regions are not "covered" by the bias charge because the edges of the potential wells are not vertical. This phenomena is called "edge effect" [4].

The increase in charge transfer inefficiency due to interfacestate trapping in surface channel devices renders them unsuitable for most applications after $10^{5} \mathrm{rad}(\mathrm{Si})$. Increasing the amount of bias charge does not appreciably improve the transfer efficiency. Surface-state trapping effects do not occur in a buried channel since the charge packet is transferred in the bulk silicon rather than at the silicon-silicon dioxide interface. The transfer inefficiency as a function of dose for surface and buried channel devices is compared in Fig. 8 [6]. The small degradation of the transfer efficiency observed in the buried channel device after large doses is probably due to gamma induced bulk trapping effects.

Interface states also act as generation recombination centers for the surface component of the dark current. The dark current density due to interface state $N_{s s}$ generation is given by [4]

$$
J_{\mathrm{DS}}=\frac{q n i}{2}\left(\pi k T \sigma_{n} v_{\mathrm{th}}\right) N_{s s}
$$

where

$$
\begin{array}{ll}
n_{i} & \text { intrinsic carrier concentration, } \\
q & \text { electronic charge, } \\
k & \text { Boltzman's constant, } \\
T & \text { absolute temperature, } \\
\sigma_{n} & \text { capture cross section for electrons, } \\
v_{\text {th }} & \text { average thermal velocity for electrons. }
\end{array}
$$

The linear relation between the interface-state density increase and the dark current increase is illustrated in Fig. 9 [10].

The thermally generated charge in a CCD limits the length of time a signal charge can be stored in the device. Dark current densities of $1000 \mathrm{nA} / \mathrm{cm}^{2}$ have been measured at $10^{6} \mathrm{rad}$ in devices having a preirradiation value of $2 \mathrm{nA} / \mathrm{cm}^{2}$ [6]. Increases of this magnitude usually prohibit application of unhardened devices after doses of $10^{5} \mathrm{rad}(\mathrm{Si})$. Ionizing radiation does not increase the number of localized regions of high dark current density (i.e., dark current spikes). 


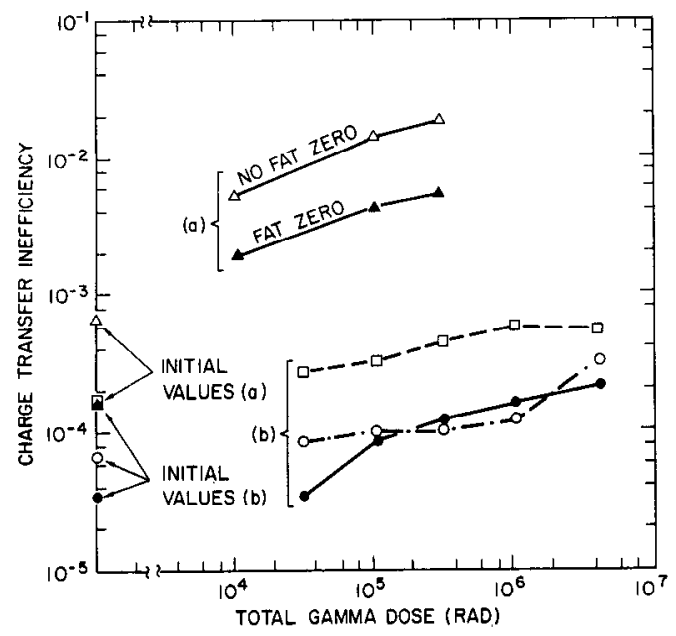

Fig. 8. Charge transfer inefficiency versus gamma dose. (a) For surface channel CCD's, (b) For buried channel CCD's.

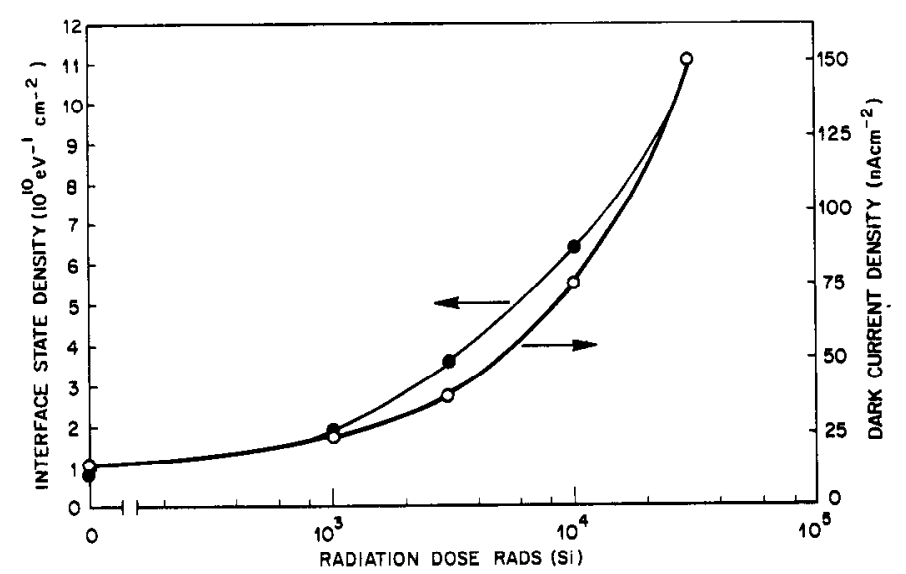

Fig. 9. Increase in dark current density and interface state density in surface channel CCD as function of dose. Data illustrate linear relation between interface state density increase and dark current increase.

\section{Transient Ionization Effects}

Charge-coupled devices are extremely sensitive to transientradiation-induced loss of stored information since they are very sensitive photosensors. The ionizing radiation creates electron-hole pairs in the bulk silicon, and those minority carriers generated within a diffusion length $L$ of the CCD potential wells will be collected. Hence, the ionizing radiation dose $\gamma$ at which the CCD becomes saturated with the radiation-induced charge is given by [12]

$$
\gamma=\frac{N_{\mathrm{FW}}}{g_{\gamma}(L+W) A_{\mathrm{bit}}}
$$

where

$$
\begin{array}{ll}
N_{\text {FW }} & \text { full-well capacity in charge carriers, } \\
W & \text { width of the depletion region, } \\
g_{\gamma} & \text { electron-hole pair generation rate constant for } \\
& \text { ionizing radiation, } \\
A_{\text {bit }} & \text { the surface area of one CCD bit. }
\end{array}
$$

For a surface channel device with $N_{\mathrm{FW}}=1.7 \times 10^{7}$ electrons, $L=150$ microns, $A_{\mathrm{bit}}=6 \mathrm{mil}^{2}$, and using $g_{\gamma}$
$=4.3 \times 10^{13}$ electron-hole pairs $/ \mathrm{cm}^{3} \mathrm{rad}(\mathrm{Si})$, the wells are predicted to saturate after a dose only $0.7 \mathrm{rad}(\mathrm{Si})$. The experimental saturation dose for this device was found to be $1 \mathrm{rad}(\mathrm{Si})$ [6]. The CCD saturation dose can be increased approximately an order of magnitude by reducing the effective depth $(L+W)$ of the collection volume associated with each CCD bit to 10 microns.

In some applications, the amount of time required to recover normal device operation after a radiation pulse is important. It has been observed that the excess charge generated in a CCD is removed at a rate which is a function of the CCD clock frequency $f_{c}$ for a given dose [13]. See Fig. 10. Additional analysis indicates a recovery time dependence on CCD well capacity $N_{\mathrm{FW}}$, since the quantity of charge carriers transported out of a CCD during a clock period is limited by the well capacity. Hence, the CCD recovery time $T_{R}$ after a pulse of ionizing radiation can be approximated by

$$
T_{R} \cong \frac{g_{\gamma} V_{\text {coll }}}{N_{\mathrm{FW}} f_{\mathrm{c}}}
$$

where $V_{\text {coll }}$ is the collection volume of one CCD bit. However, the data in Fig. 10 show that the recovery time does not increase linearily with radiation pulse dose. This effect can probably be attributed to electron-hole pair recombination and direct carrier removal through the output diode for large overload doses.

Device burnout can be prevented by simply current limiting the power supply leads to the CCD diodes. This technique has been employed successfully for dose rates up to $10^{11} \mathrm{rad}$ (Si)/s [6] .

Implicit in this pulsed radiation saturation discussion has been the assumption that the radiation dose is delivered in a period of time that is much shorter than the time a given potential well exists in the CCD. The background radiation level that results in CCD-well saturation depends on the time a given depletion region exists in the CCD. For a device operating in the integration mode, the wells fill with charge primarily during the integration time, while in the continuous mode of operations, the time a potential well remains in the device is given by the product of the device length in (bits) and the shift register clock period. Surface channel devices have a higher saturation level since in general they have a larger charge handling capacity per unit gate area.

\section{DISPLACEMENT DAMAGE EFFECTS}

Fast neutrons create bulk trapping centers in silicon which cause a decrease in transfer efficiency primarily in buried channel devices and an increase in the bulk component of the dark current density in both surface and buried channel structures. The transfer inefficiency $\epsilon$ in a buried channel CCD for a single level of traps is related to the bulk trap density $N_{t}$ by [14], [15]

$$
\epsilon=\frac{V_{\mathrm{SIG}}}{N_{s}} N_{t} e^{-T_{t} / \tau i}\left(1-e^{-T_{E} / \tau i}\right)
$$

where

$V_{\text {SIG }}$ volume occupied by the signal charge packet, $N_{s}$ number of signal charges in volume $V_{\text {SIG }}$, 


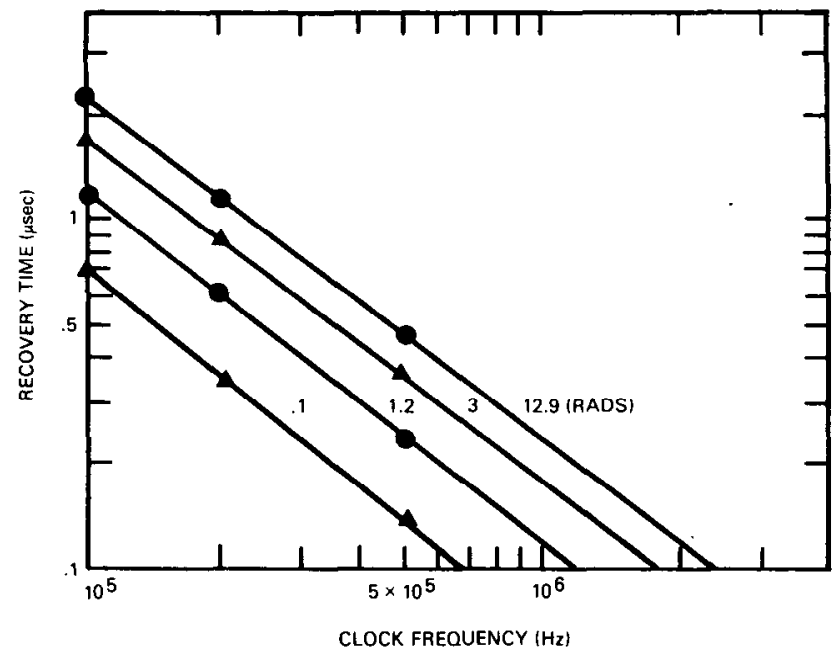

Fig. 10. CCD recovery time as function of clock frequency and pulse dose.

$T_{t} \quad$ time available for charge transfer between adjacent electrodes,

$\tau_{i} \quad$ bulk trap emission time constant,

$T_{E} \quad$ time the bulk traps have to empty between signal charge packets.

The measurement of the transfer inefficiency as a function of the time the bulk traps are allowed to empty is known as the "double-pulse" technique. The fit of the transfer inefficiency equation to the double-pulse data before neutron irradiation is shown in Fig. 11 [16].

Increased trapping effects in neutron irradiated $n$-buried channel CCD's are insignificant for fluences less than $10^{11} \mathrm{n} / \mathrm{cm}^{2}(\sim 15 \mathrm{MeV}$ average) [16]. The linear transfer inefficiency increase in the $10^{11}-10^{13} \mathrm{n} / \mathrm{cm}^{2}$ fluence range is shown in Fig. 12. After $10^{13} \mathrm{n} / \mathrm{cm}^{2}(\sim 15 \mathrm{meV}$ average) the transfer efficiency at $295 \mathrm{~K}$ was reduced to 0.992 , making the devices unsuitable for most applications. The degradation obserced after $15-\mathrm{MeV}$ neutron bombardment is expected to be 2.5-3 times greater than the value for a $1-\mathrm{MeV}$ equivalent neutron fluence [17].

Neutron irradiation produces several trapping levels of unequal density in silicon. Three distinct trap levels have been observed from 77-300 K by use of the double-pulse technique [18]. The energy level $\left(E_{c}-E_{t}\right)$ and the creation rate $\left(\Delta N_{t} / \Delta \phi\right)$ of the bulk traps are given in Table I. Similar trapping levels have been observed in other types of irradiated silicon devices [19].

Bulk traps are created during neutron bombardment in both surface and buried channel CCD structures. However, the volume occupied by a charge packet in a surface channel device is more than an order of magnitude smaller than the volume in a buried channel structure. Consequently, the transfer efficiency in a surface channel is less sensitive to increases in bulk trap density since the charge packets interact with fewer bulk traps. See Fig. 13 [6] .

While 50 percent of the fast neutron energy is deposited in silicon via displacement damage, the fraction for gamma rays is much smaller. The bulk trap creation rate for $1-\mathrm{MeV}$ photon/ $/ \mathrm{cm}^{2}$ is only $1 \times 10^{-3} \mathrm{~cm}^{-1}$ [20]. Hence, bulk

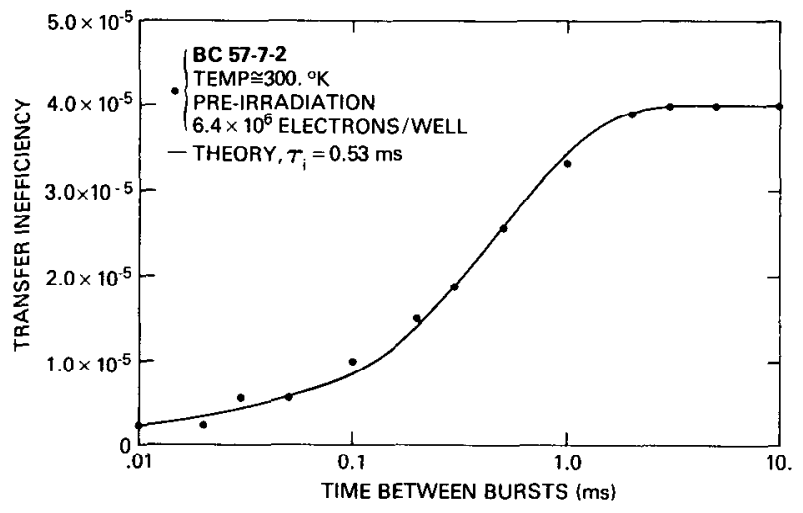

Fig. 11. Double-pulse data for unirradiated $n$-buried channel CCD and fit of discrete bulk trap level theory (solid line) to data. Bulk trap emission time constant obtained from this fit was $0.53 \mathrm{~ms}$ at $300 \mathrm{~K}$.

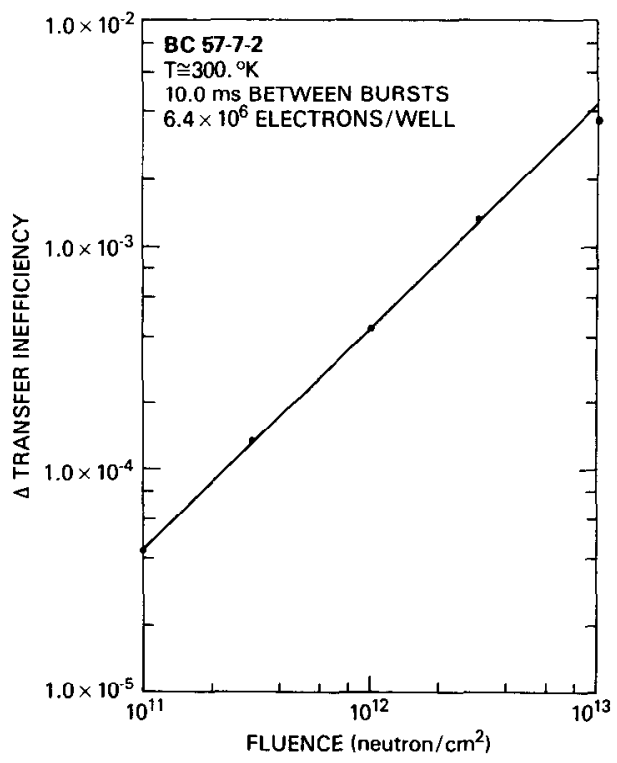

Fig. 12. Change in transfer inefficiency at $300 \mathrm{~K}$ as function of neutron fluence, illustrating linear relation between increase in transfer inefficiency due to bulk trapping and neutron fluence.

TABLE 1

Bulk Trap Energy Levels and Creation Rates

\begin{tabular}{ccc}
\hline Leve1 & $\begin{array}{c}\left(\mathrm{E}_{\mathrm{c}}-\mathrm{E}_{\mathrm{t}}\right) \\
\mathrm{eV}\end{array}$ & $\begin{array}{c}\Delta \mathrm{N}_{\mathrm{t}} / \Delta \phi \\
\mathrm{cm}^{-1}\end{array}$ \\
$\mathrm{~N}-1$ & 0.14 & 1.1 \\
$\mathrm{~N}-2$ & 0.23 & $\sim 0.8$ \\
$\mathrm{~N}-3$ & 0.41 & 7.0 \\
\hline
\end{tabular}




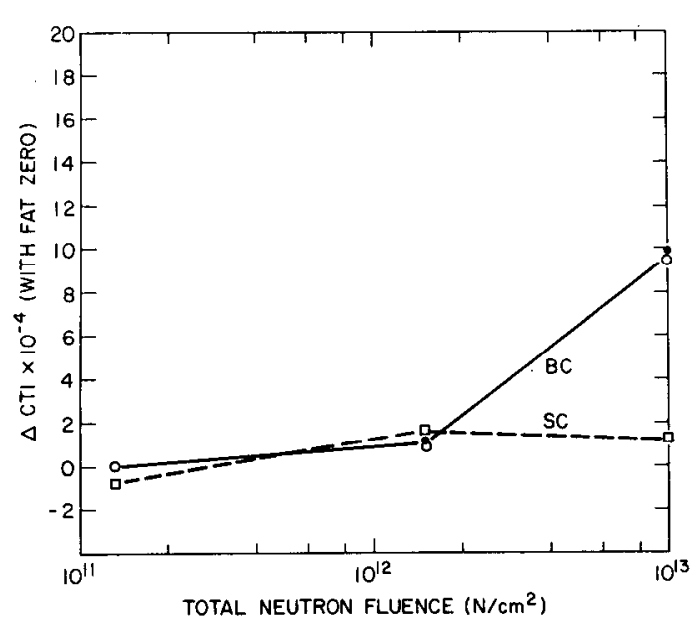

Fig. 13. Change in charge transfer inefficiency (CTE) with neutron fluence ( $1 \mathrm{MeV}$ equivalent) for typical surface and buried channel CCD's.

damage effects due to gamma irradiation are not significant for doses less than $10^{6} \mathrm{rad}(\mathrm{Si})\left(2 \times 10^{15} 1 \mathrm{MeV}\right.$ gammas $\left./ \mathrm{cm}^{2}\right)$. The carrier removal rates for $1.7 \mathrm{MeV}$ electrons is in the $0.2-1.0 \mathrm{~cm}^{-1}$ range [21]

Little progress has been made in the area of neutron hardening of buried channel CCD's. An obvious technique would be to fabricate a thinner but more heavily doped buried channel so that the signal charge packet would interact with a small number of bulk traps, thereby reducing the transfer efficiency degradation.

The bulk trap level located near mid gap, $E_{c}-E_{t}=0.41$ $\mathrm{eV}$, acts as a bulk recombination generation center for dark current in both surface and buried channel devices. An approximate linear increase in dark current density in the $10^{11}-10^{13} \mathrm{n} / \mathrm{cm}^{2}$ range is shown in Fig. 14 for an $n$-buried channel device [16]. The surface component of the dark current density did not increase since the total dose equivalence of the $10^{13} \mathrm{n} / \mathrm{cm}^{2}$ fluence was only approximately $10^{4} \mathrm{rad}$ (Si). [22]

A linear relation between the dark current density increase and the neutron fluence is predicted from a simple consideration of the radiation dependence of the minority carrier lifetime $\tau[12]$ :

$$
\frac{1}{\tau}=\frac{1}{\tau_{0}}+K \phi
$$

where

$$
\begin{array}{ll}
\tau_{0} & \text { preirradiation minority carrier lifetime, } \\
K & \text { minority carrier lifetime degradation constant, } \\
\phi & \text { neutron fluence. }
\end{array}
$$

The bulk component of the dark current density is given by

$$
J_{D}=\frac{q n_{i} d}{2 \tau}
$$

where

$q$ electronic charge,

$n_{i} \quad$ intrinsic carrier concentration,

$d$ depth of the depletion region.

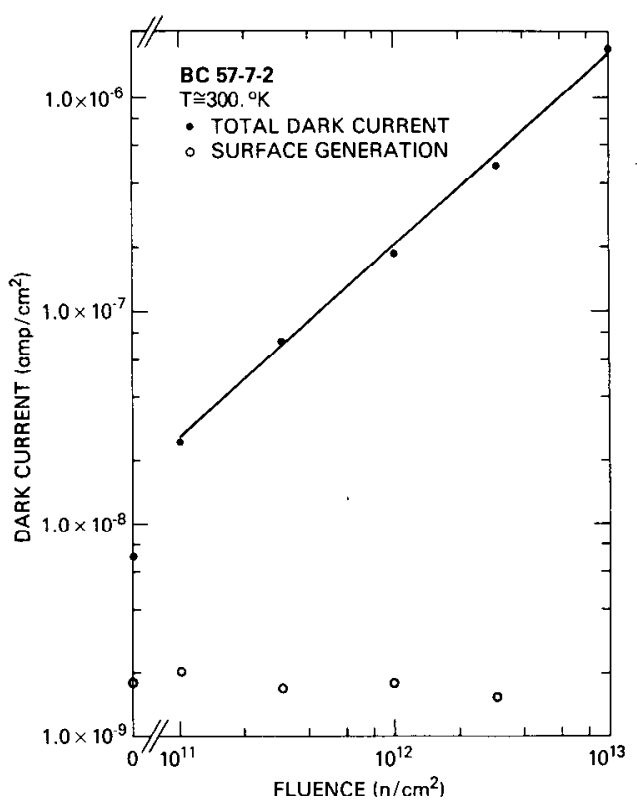

Fig. 14. Dark current density at $300 \mathrm{~K}$ as function of neutron fluence, illustrating linear relation between $J_{D}$ and neutron fluence.

Hence, the increase in the bulk component of the dark current density is related to the neutron fluence by

$$
\Delta J_{D}=\frac{q n_{i} d}{2} K \phi
$$

The value of $K$ obtained from the data shown in Fig. 14 is $K=$ $2.3 \times 10^{-6} \mathrm{~cm}^{2} \mathrm{~s}^{-1}$. The values of $K$ quoted in the literature are in the $10^{-6} \mathrm{~cm}^{2} \mathrm{~s}^{-1}$ range [23].

\section{CCD SURFACE DAMAGE HARDENING}

\section{Structural Optimization}

The earliest work on total dose irraditation effects in CCD's identified the major failure mechanisms peculiar to several device structures since a standard CCD design had not then emerged [10]. The objective was to identify those failure mechanisms inherent in a particular structure so that the optimum design for total dose radiation hard CCD's could be determined. In particular the following were found.

1) A buried channel structure should be used. The charge transfer efficiency in a buried channel device, contrary to surface channel CCD's, is not degraded by an increase in the interface-state trap density after irradiation.

2) An $n$-buried channel CCD structure is preferred. The flatband voltage shift for a given oxide structure during irradiation is minimized when the gate electrodes are negative with respect to the channel potential.

3) The $n$-buried channel CCD output diode should be capable of being reversed biased to a voltage which will allow the channel to remain depleted after irradiation. The negative flatband voltage shift in an $n$-buried channel structure causes the buried channel to be driven out of depletion. A few volts of flatband shift can be automatically accommodated by biasing the output diode to a value several volts in excess of the preirradiation bias required to deplete the buried channel. 
4) The design should use a planar channel insulator (no stepped oxide) and only one type of electrode material. This is necessary to eliminate differences in the flatband voltage shift under adjacent electrodes, since such differences can reduce or eliminate potential barriers which can result in reduced well capacity and increased charge transfer inefficiency.

5) The use of undoped polysilicon for interelectrode isolation should be avoided. Experience on two different types of devices has shown that total doses of 1 to $3 \times 10^{4} \mathrm{rad}(\mathrm{Si})$ cause channeling in the isolation regions with resulting deterioration in device performance [10].

6) The input structure should be compatible with the operation of a threshold insensitive input technique if an analog input is required.

\section{Hard Oxide Technology}

Structural optimization alone is not sufficient to enable CCD's fabricated using standard gate oxide techniques to satisfy the total dose radiation requirements for most space and strategic applications. The approach taken in the development of a radiation-hard insulator for CCD's was to modify the process used in the fabrication of radiation-hard CMOS devices. [24], [25] An outline of the fabrication steps for a radiationhard $n$-buried channel process is shown in Fig. 15 [26]. Some of the process variations which have significant effect on the CCD hardness are the following.

1) A field oxide is grown and stripped to remove the damaged surface in the region where the gate oxide is to be grown.

2) The number of high-temperature processing steps required after the growth of the gate oxide is minimized by performing source-drain diffusions and bulk-state gettering prior to the growth of the gate oxide. The choice of a $925^{\circ} \mathrm{C}$ pyrogenic oxide was made for the same reason (i.e., faster growth rate).

3) The buried channel is implanted before the gate oxide is grown. Ion implantation through a radiation-hard oxide may result in reduced radiation hardness even after the implantation produced flatband voltage shift has been annealed.

4) The oxide thickness under all CCD gates is made approximately equal to minimize differences in flatband voltage shift after irradiation. The oxide is made thin as is feasible in order to reduce flatband voltage shift.

5) Eliminate the use of an electron gun for the aluminum metallization. The $\mathrm{X}$-ray radiation dose absorbed during a typical $e$-beam process $\left(10^{6} \mathrm{rad}(\mathrm{Si})\right)$ seriously degrades oxide radiation hardness for negative gate to channel bias during subsequent radiations [27].

Both surface and buried channel shift registers have been fabricated using the hard-oxide process. [25], [28] These devices can be operated after $10^{6} \mathrm{rad}(\mathrm{Si})$ with the preirradiation clock and bias voltages. The postirradiation values for the $n$-buried channel device parameters, listed in Table II, are acceptable for many radiation environment applications [26]

The transfer efficiency in surface channel devices fabricated with this radiation-hard oxide was seriously degraded for doses greater than $10^{5} \mathrm{rad}(\mathrm{Si})[25]$. See Table III. A severe increase in interface-state trapping required the use of a 50 percent bias charge to obtain a transfer efficiency of $0.992 \mathrm{after} 10^{6} \mathrm{rad}$

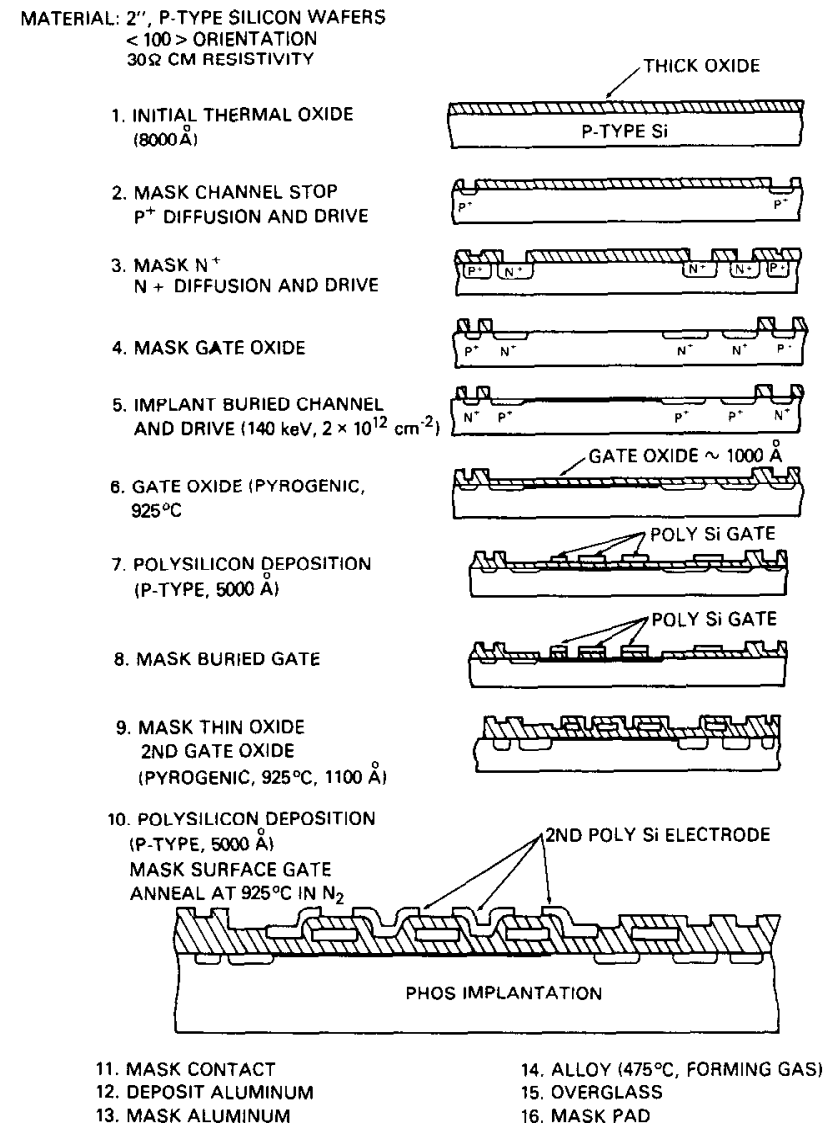

Fig. 15. Outline of optimum radiation-hard $n$-buried channel CCD fabrication process.

(Si). The dark current density and threshold voltage shift after $10^{6} \mathrm{rad}(\mathrm{Si})$ were approximately equal to the values observed in the buried channel structure.

It should be noted that the pyrogenic $\mathrm{CCD}$ hard oxide process described is insensitive to total dose radiation effects only for negative gate to channel bias (i.e., $p$-surface channel and $n$-buried channel). The flatband shift for positive gate to channel bias, shown in Fig. 16, is quite large. Bulk silicon damage effects in neutron-irradiated radiation hard devices do not differ from those in unhardened structures.

\section{IRRADIATION EFFECTS AT CRYOGENIC TEMPERATURES}

CCD's are being considered for use in space and infrared imaging systems both as infrared (IR) detectors and as signal processors for IR focal plane arrays at low temperatures. However, several studies have shown that charge buildup in $\mathrm{SiO}_{2}$ is more rapid in devices irradiated at low temperatures. [29], [30] Large flatband voltage shifts are observed during $77 \mathrm{~K}$ irradiation, in oxides which are radiation hard at room temperature [31]. The increased charge trapping effects at low temperatures in the CCD radiation hard oxide shown in Fig. 17 make these devices unsuitable for most $77 \mathrm{~K}$ applications after a dose of $5 \times 10^{4} \mathrm{rad}(\mathrm{Si})$ [32]. Several techniques (alternate insulators, metal nitride oxide structures, and aluminum implanted oxides) have been suggested for improving the low-temperature radiation behavior of MIS structures [33], but their use in CCD fabrication has not been reported. 
TABLE II

\begin{tabular}{|c|c|c|}
\hline & Radiation Hard $n$-Buried Channel CCD & Parameters \\
\hline & Pre-Irradiation & $1 \times 10^{6} \operatorname{rads}(S i)$ \\
\hline CTE & 0.99999 & 0.9999 \\
\hline$J_{D}$ & $5 \mathrm{nA} / \mathrm{cm}^{2}$ & $140 \mathrm{nA} / \mathrm{cm}^{2}$ \\
\hline We11 Capacity & $3 \times 10^{6} e^{-}$ & $2.25 \times 10^{6} \mathrm{e}^{-}$ \\
\hline Threshold Shift & - & $-1.8 \mathrm{~V}$ \\
\hline
\end{tabular}

TABLE III

\begin{tabular}{lrl}
\hline & Radiation Hard p-Surface Channel CCD Parameters \\
\hline & Pre-Irradiation & $1 \times 10^{6}$ rads (Si) \\
CTE & $0.9997 \quad 20 \% \mathrm{BC}$ & $0.99250 \% \mathrm{BC}$ \\
$\mathrm{J}_{\mathrm{D}}$ & $20 \mathrm{nA} / \mathrm{cm}^{2}$ & $100 \mathrm{nA} / \mathrm{cm}^{2}$ \\
Threshold Shift & - & $-2.0 \mathrm{~V}$
\end{tabular}

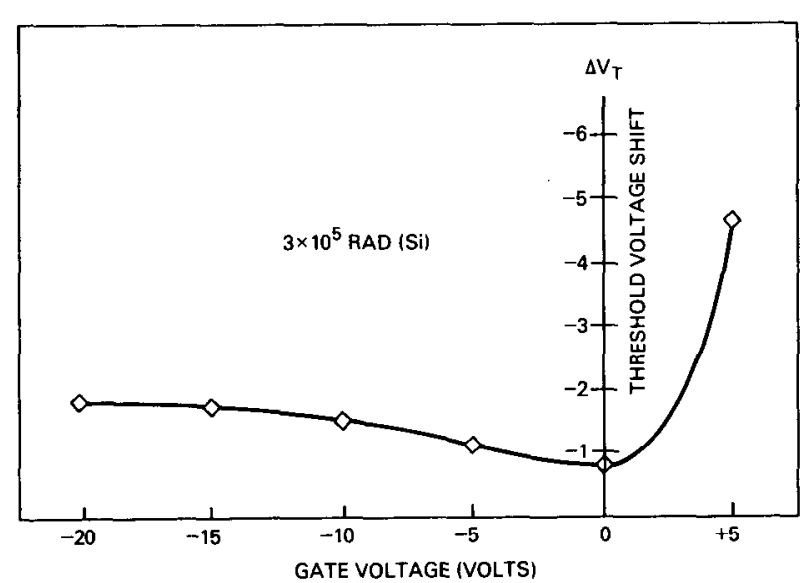

Fig. 16. Threshold voltage shift as function of gate bias, illustrating radiation sensitivity of $C C D$-hard oxide for positive gate bias during irradiation.

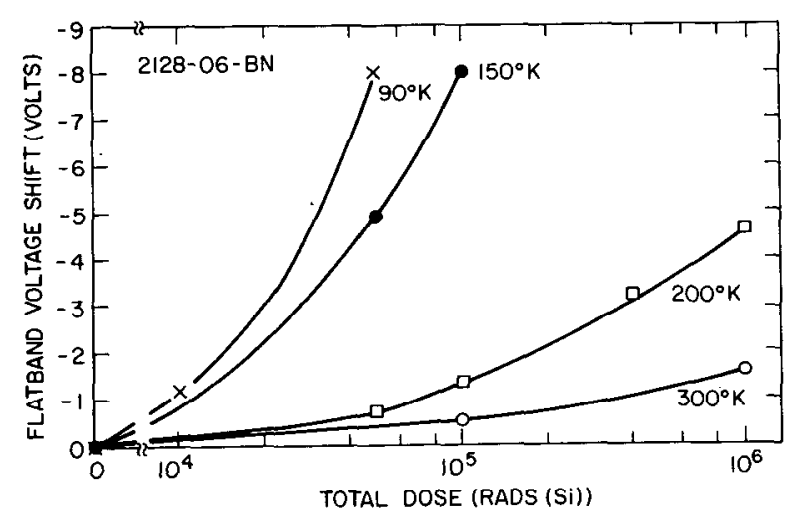

Fig. 17. Flatband voltage shift for $\mathrm{CCD}$ radiation hard oxide as function of dose at several temperatures, illustrating increased oxide charge trapping effects at low temperatures.
The holes generated in the oxide during a liquid nitrogen temperature irradiation are trapped almost immediately producing a nearly uniform density of positive charge in the oxide. The resultant flatband voltage shift is proportional to the oxide thickness squared and has a value of $-2.0 \mathrm{~V} / 10^{4} \mathrm{rad}$ (Si) for a $1000 \AA$ oxide if all the generated holes are trapped uniformly [34]. The fraction of the holes trapped is independent of gate voltage polarity for small doses, but is a function of the electric field strength in the oxide. [35]

Several techniques have been employed to anneal the excess flatband voltage shift observed in devices irradiated at low temperatures. These include: photodepopulation of the traps, field-aided emission of holes from traps, and thermal annealing [36]. Room temperature radiation hard oxides show some recovery even at $85 \mathrm{~K}$, but the annealing process is accelerated at temperatures greater than $125 \mathrm{~K}$ [37].

The excess low-temperature irradiation threshold voltage shift observed in the room-temperature radiation-hard CCD was annealed by warming the device to room temperature [32]. After recooling the CCD, threshold voltage shift was approximately equal to the shift which would have been observed if the devices had been irradiated at $300 \mathrm{~K}$ and then cooled. The input gate threshold voltage shift for several irradiation-anneal cycles is shown in Fig. 20. The dotted lines indicate the threshold voltage shift during the thermal annealing process (approximately $20 \mathrm{~min}$ were required to warm the dewar). The first peak in Fig. 20 corresponds to a dose of $3 X$ $10^{4} \mathrm{rad}$ at $85 \mathrm{~K}$. The second and third peaks are the shifts for incremental doses of $5 \times 10^{4}$ rad at $85 \mathrm{~K}$. Normal CCD clock and bias voltages were applied to the device during the irradiation-annealing sequence. The residual shift at liquid nitrogen temperatures increased to $-0.5 \mathrm{~V}$ after $1.3 \times 10^{5} \mathrm{rad}$ as expected from $300 \mathrm{~K}$ irradiation results. A residual shift of up to 


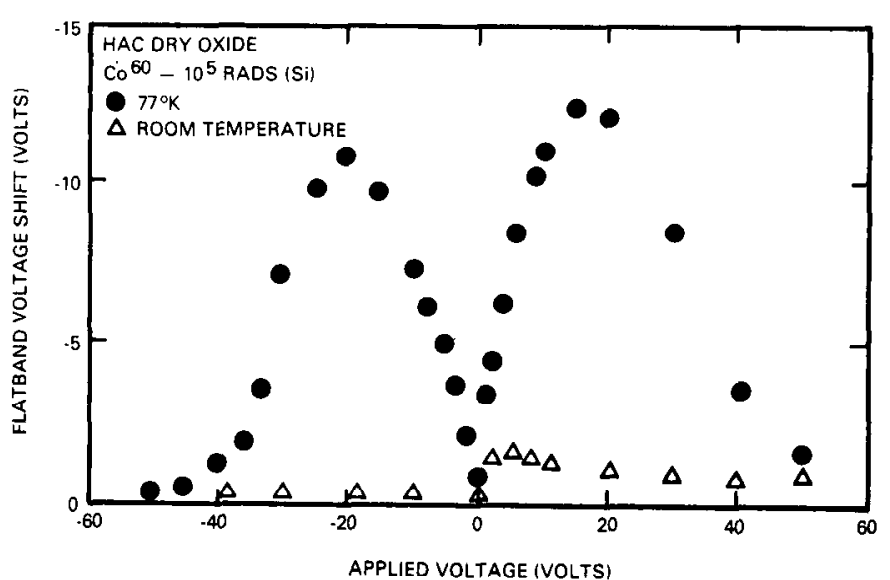

Fig. 18. Flatband voltage shift at $10^{5} \mathrm{rad}(\mathrm{Si})$ as function of applied voltage, illustrating electric field dependence of flatband voltage shift at $77 \mathrm{~K}$.

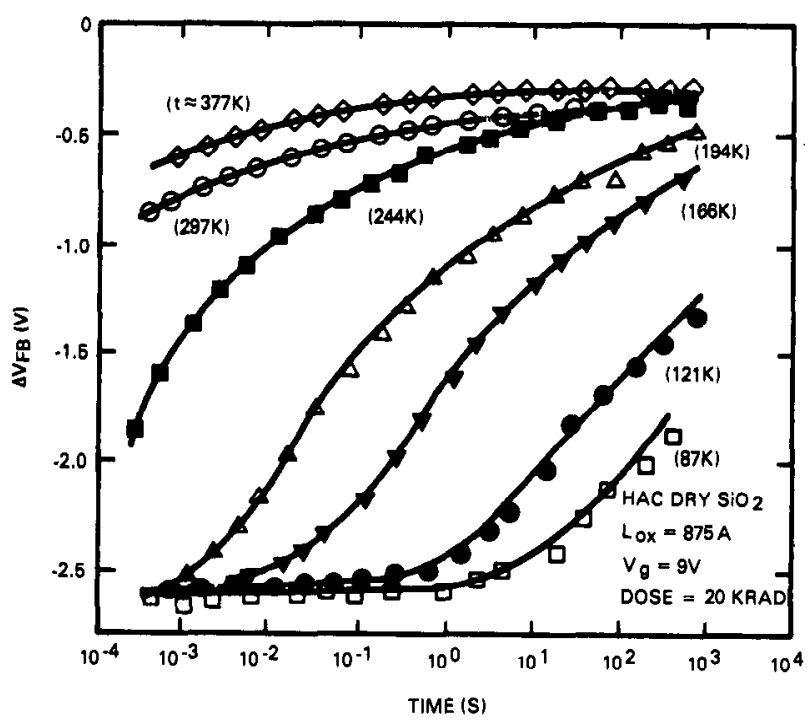

Fig. 19. Flatband voltage shift as a function of time after a $20 \mathrm{krad}$ radiation pulse, illustrating annealing of the flatband voltage shifts at various temperatures.

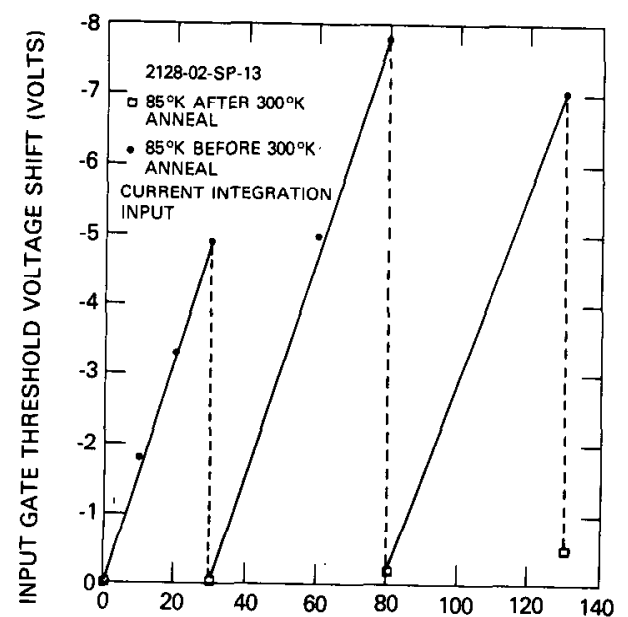

Fig. 20. Radiation-hard $\mathrm{CCD}$ input gate threshold voltage shift at $85 \mathrm{~K}$ as function of dose and $300 \mathrm{~K}$ annealing. ( $p$-surface channel).
$-5 \mathrm{~V}$ might be expected for a typical nonhardened oxide subjected to the same irradiation-annealing cycle.

The flatband voltage shift in the CCD hard oxide during irradiation at $4.2 \mathrm{~K}$ has been recently been found to be identical to the shift for $77 \mathrm{~K}$ irradiation [38]. However, it has also been shown that the MNOS approach offers considerable improvement in the radiation hardness of capacitors irradiated at $77 \mathrm{~K}$ [39]. This technique may be useful for radiation-hard devices at $4.2 \mathrm{~K}$.

The limited amount of experimental data available suggest that the permanent bulk silicon damage caused by fast neutrons in CCD's irradiated at $80 \mathrm{~K}$ and $294 \mathrm{~K}$ is similar [16] . The energy and density of the $N-1$ trap level was independent of irradiation temperature ( $80 \mathrm{~K}$ or $294 \mathrm{~K}$ ) and irradiation bias. However, the transfer inefficiency in a buried channel device will change as the temperature is varied since the bulk trap emission time constant is temperature dependent [14]. The shift of the double-pulse transfer inefficiency curve to longer times between bursts with reduction in $\mathrm{CCD}$ operating temperature is shown in Fig. 21. [18] At $80 \mathrm{~K}$, the emission time constant for the trap level $(N-3)$, which controls the transfer loss at $294 \mathrm{~K}$, has increased to a time much longer than $1 \mathrm{~s}$. Consequently, these traps remain filled during the usual times involved in the double-pulse measurement and do not contribute to loss of signal charge in the CCD. The post neutron irradiation transfer loss at $80 \mathrm{~K}$ is dominated by the lower density $N-1$ trap level. The reduced transfer inefficiency at $80 \mathrm{~K}$ is compared to the $294 \mathrm{~K}$ values in Fig. 22 [16].

\section{SUMMARY}

The previous sections of this paper have presented an overview of the current state-of-the-art of radiation effects and hardening techniques. The key points presented are listed below.

1) CCD's are sensitive to both surface and bulk damage effects due to radiation. Devices fabricated using standard com. mercial gate oxide technologies are unable to satisfy the total dose requirements for most space and strategic requirements.

2) An $n$-buried channel device is the least radiation sensitive structure for total ionizing dose effects.

3) The neutron induced degradation of the charge transfer efficiency is greater in buried channel devices than in surface channel devices.

4) Only a limited increase in the total dose radiation tolerance of CCD's can be achieved by means of structural and operational considerations alone. A radiation-hard oxide technology is required to satisfy system radiation requirements.

5) CCD's are extremely sensitive to transient upset effects. Increased tolerance can be achieved by thinning. Device burnout is prevented by current limiting the power supplies.

6) An megarad-hardened $n$-buried channel hard CCD technology has been developed, and simple linear radiation hard shift registers have been fabricated with the optimized structure.

7) Total ionizing dose effects are more severe for irradiation at $77 \mathrm{~K}$. Ordinary room-temperature radiation hardening techniques do not apply for irradiation at liquid nitrogen tempera- 


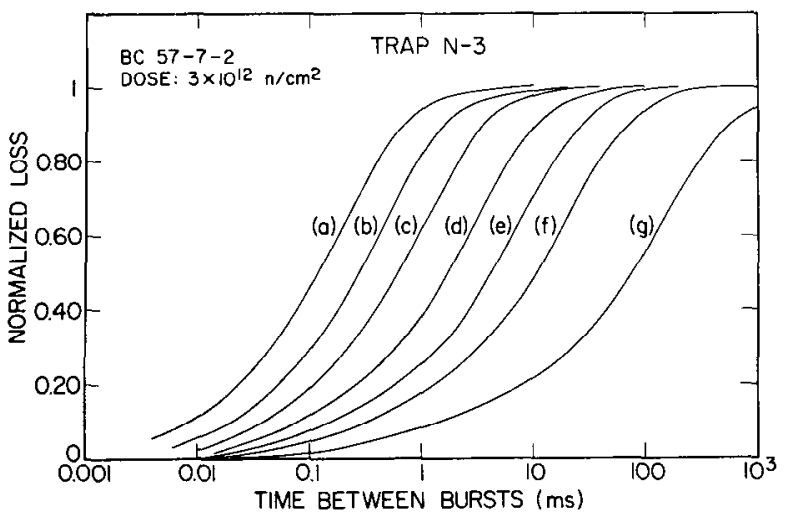

Fig. 21. Normalized double-pulse data (i.e., $\left(\epsilon / \epsilon_{\max }\right)$ for $n$-buried channel device irradiated to $3 \times 10^{12} \mathrm{n} / \mathrm{cm}^{2}$, illustrating shift in double-pulse curve to longer times (i.e., position (a) to position (g)) as temperature is changed from $262 \mathrm{~K}$ to $199 \mathrm{~K}$.

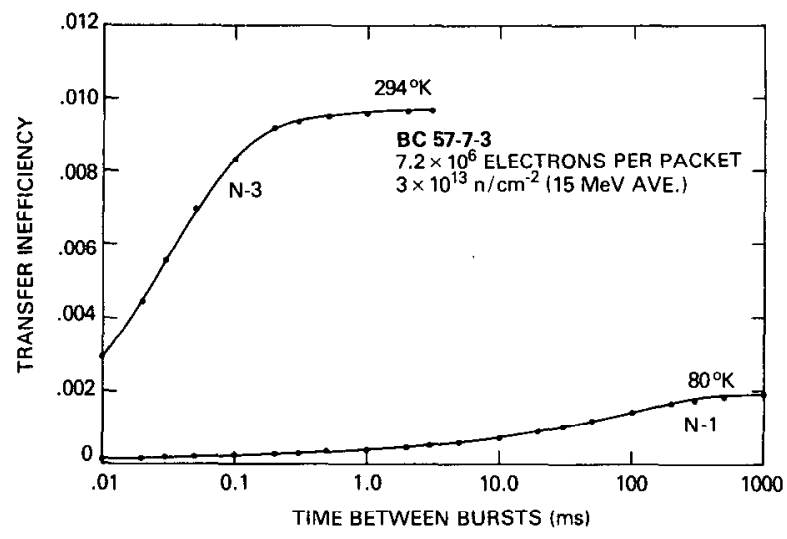

Fig. 22. Double pulse curves at $294 \mathrm{~K}$ and $80 \mathrm{~K}$ for CCD irradiated to $3 \times 10^{13} \mathrm{n} / \mathrm{cm}^{2}$. At $80 \mathrm{~K}, \mathrm{~N}-1$ has smaller density than trap level affective at $294 \mathrm{~K}$. Hence, charge transfer inefficiency is smaller at $80 \mathrm{~K}$.

tures. However, several techniques have been proposed to solve this problem.

8) The degradation of CCD parameters in neutron-irradiated buried channel CCD's are less severe at $77 \mathrm{~K}$ than at $300 \mathrm{~K}$.

\section{ACKNOWLEDGMENT}

The author thanks N. S. Saks, W. D. Baker, and D. F. Barbe for many discussions on radiation effects in CCD's and for reviewing this paper.

\section{REFERENCES}

[1] B. L. Gregory and C. W. Gwyn, "Radiation Effects on Semiconductor Devices," Proc. IEEE, 62, 1264 (1974).

[2] D. F. Barbe, "Imaging Devices Using the Charge-Coupled Concept," Proc. IEEE, 63, 38 (1975).

[3] J. D. E. Beynon, "The Basic Principles of Charge-Coupled Devices," Microelectronics Journal, 7, No. 2., 7 (1975).

[4] C. H. Sequin, and M. F. Tompsett, "Charge Transfer Devices," Academic Press, New York 1975.

[5] D. F. Barbe, "Charge Integration and Storage in MOS Photosensors," ch. 3 pp. 219-231 in Solid State Imaging Eds. P. G. Jespers, F. Van De Wiele and M. H. White, Leydon, Netherlands, Noordhoff, 1976.

[6] G. A. Hartsell, "Radiation Hardness of Surface and Buried Channel CCD's," Proc. International Conf. on the Appl. of $C C D$ 's, 375 (1975).

[7] C. H. Sequin, and A. M. Mohsen, "Linearity of Electrical Charge
Injection into Charge Coupled Devices", IEEE J. Solid-State Circuits, SC-10, 81 (1975).

[8] J. M. Killiany and W. D. Baker, "Limitations of a ThresholdInsensitive CCD Input Technique in a Total Dose Radiation Environment", Proc. International Conf. on the Appl. of CCD's, 369 (1975).

[9] D. F. Barbe, J. M. Killiany, and H. L. Hughes, "Effects of Gamma Radiation in Charge-Coupled Devices," Appl. Phys. Lett., 23, 400 (1973).

[10] J. M. Killiany, W. D. Baker, N. S. Saks, and D. F. Barbe, "Effects of Ionizing Radiation on Charge-Coupled Device Structures," IEEE Trans. Nuc. Sci, NS-21, No. 6, 193 (1974).

[11] J. E. Carnes, and W. F. Kosonocky, "Charge-Coupled Devices and Applications," Solid State Technol., 17, 67 (1974).

[12] R. A. Williams and R. D. Nelson, "Radiation Effects on ChargeCoupled Devices," IEEE Trans. Nuclear Science, NS-22, pp. 2639-2644, (1975).

[13] W. Shedd and B. Buchanan, "Transient Radiation Effects in CCD's," IEEE Trans. Nuc. Sci., NS-23, 1636 (1976).

[14] A. M. Mohsen and M. F. Thompsett, "The Effects of Bulk Traps on the Performance of Bulk Channel CCD's, IEEE Trans. Elec. Dev., ED-21, p. 701, Nov. 1974.

[15] R. W. Broderson, and S. P. Emmons, "The Measurement of Noise in Buried Channel CCD's", Proc. Int. Conf. Appl. CCDs (29-31 Oct. 1975), pp. 331-349.

[16] N. S. Saks, J. M. Killiany, and W. D. Baker, "Effects of Neutron Irradiation on the Characteristics of a Buried Channel CCD at $80^{\circ} \mathrm{K}$ and $295^{\circ} \mathrm{K}$," Proc. NASA-JPL Conf. CCD Tech. and Applications, Washington, D.C., Dec. 1976.

[17] E. C. Smith, D. Binder, P. A. Compton, and R. I. Wilbur, "Theoretical and Experimental Determinations of Neutron Energy Deposition in Silicon," IEEE Trans. Nuc. Sci., NS-13, No. 6, 11 (1966).

[18] N. S. Saks, "Investigation of Bulk Electron Traps Created by Fast Neutrons Irradiation in a Buried Channel CCD," IEEE Trans. Nuc. Sci. NS-24, 2153 (1977).

[19] J. W. Walker and C. T. Sah, "Properties of $1.0 \mathrm{MeV}$ ElectronIrradiated Defect Centers in Silicon", Phy. Rev. B, 7, p. 4587, May 1973.

[20] O. L. Curtis, and J. R. Srour, "Recombination Within Disordered Regions: Influence of Barrier Height on Recombination Rate and Injection Level Effects", IEEE Trans. Nuc. Sci. NS-20, No. 6, (1973).

[21] H. J. Stein and R. Gereth, "Introduction Rates of Electrically Active Defects in n-and p-type Silicon by Electrically Active Defects," J. Appl. Phys., 31, 2890 (1968).

[22] R. A. Poll, "Electronic System Hardening Approaches", IEEE Trans. Nucl. Sci., NS-18, pp. 100-103, Dec. 1971.

[23] R. R. Holmes, "Carrier Removal in Neutron Irradiated Silicon," IEEE Trans. Nuc. Sci., NS-17, 137, Dec. (1970).

[24] G. F. Derbenwick and B. L. Gregory, "Process Optimization of Radiation-Hardened CMOS Integrated Circuits," IEEE Trans. Nuc. Sci., NS-22, 2151, (1975).

[25] C. P. Chang, "Radiation Hardened Surface Channel CCD's," IEEE Trans. Nuc. Sci., NS-23, 1639 (1976).

[26] C. P. Chang, and K. G. Aubuchon, Final Report, Contract Number N00173-77-C-0158, Hughes Aircraft Company, Newport Beach, CA, July, 1978.

[27] S. Mayo, K. F. Galloway, and T. F. Leedy, "Radiation Dose Duc to Electron-Gun Mettalization Systems" IEEE Trans. Nuc. Sci., NS-23, 1875 (1976).

[28] C. P. Chang, "Radiation Effects in N-Buried Channel CCD's Fabricated with a Hardened Process," IEEE Trans. Nuc. Sci., NS-24, 2190 (1977).

[29] K. L. Nielsen and D. K. Nichols, "Total Dose Effects of Ionizing Radiation on MOS Structures at $90^{\circ} \mathrm{K}$," IEEE Nuc. Sci., NS-20, No. 6, 319 (1973).

[30] R. C. Hughes, "Hole Mobility and Transport in Thin $\mathrm{SiO}_{2}$ Films," Appl. Phys. Lett. 26, No. 8, (1975).

[31] H. F. Boesch, Ir., F. B. McL ean, J. M. McGarrity, G. A. Ausman, Jr., "Hole Transport and Charge Relaxation in Irradiated $\mathrm{SiO}_{2}$ MOS Capacitors", IEEE Trans. Nuc. Sci., NS-22, 2163 (1975).

[32] J. M. Killiany, "Low Temperature Radiation Damage Effects in a Room Temperature Radiation-Hard Surface Channel CCD," IEEE Trans. Nuc. Sci., NS-24, 2194 (1977).

[33] J. R. Srour, S. Othmer, O. L. Curtis, Jr., and K. Y. Chin, "Radiation-Induced Charge Transport and Charge Buildup in 
$\mathrm{SiO}_{2}$ Films at Low Temperatures," IEEE Trans. Nuc. Sci., NS-23, 1513 (1976).

[34] H. H. Sander and B. L. Gregory, "Unified Model of Damage Annealing in CMOS from Freeze-In to Transient Annealing", IEEE Trans. Nuc. Sci., NS-22, 2157 (1975).

[35] J. R. Srour and K. Y. Chiu, "MOS Hardening Approaches for Low Temperature Applications," IEEE Trans. Nuc. Sci. NS24, 2141 (1977).

[36] E. Harari, S. Wang, and B. S. H. Royce, "Low-Temperature Irradiation Fffects is $\mathrm{SiO}_{2}$ Insulated MIS Devices," I. Appl. Phys., 46, 1310 (1975).
[37] F. B. McLean, H. E. Boesch, Jr., and J. M. McGarrity, "Hole Transport and Rccovery Charactcristics of $\mathrm{SiO}_{2}$ Gatc Insulators," IEEE Trans. Nuc. Sci, NS-23, 1506 (1976).

[38] S. Othmer and J. R. Srour, "Charge Buildup in Irradiated $\mathrm{SiO}_{2}$ Films at $4^{\circ} \mathrm{K}$," presented at the IEEE Annual Conf. on Nuclear and Space Radiation Effects, Albuquerque, N.M. July 18-21, 1978.

[39] N. S. Saks, "Response of MNOS Capacitors to Ionizing Radiation at $80^{\circ} \mathrm{K}$," presented at the IEEE Annual Conf. on Nuclear and Space Radiation Effects, Albuquerque, N.M. July 18-21. 1978.

\title{
Five-Year Life Test Data on Pressure (10 $000 \mathrm{lbf} /$ in $\left.^{2}\right)$ Tolerant Electronic Components
}

\author{
ROBERT A. MARQUARDT
}

\begin{abstract}
A program is described where long-term reliability data on contemporary electronic components under a fluid pressure environment were accumulated. Resistors, capacitors, diodes, integrated circuits, inductors, and preamplifier modules that showed excellent short-term immunity to pressure were pressure-cycled five times and stored in silicone oil at $10000 \mathrm{lbf} / \mathrm{in}^{2}$ (deep ocean pressure) for five years. The test results of these six classes of components $(750$ individual components) indicate that the high-pressure oil is not a harsh environment if the components and oil are properly selected and screened.
\end{abstract}

\section{INTRODUCTION}

$\mathbf{E}$ MPHASIS on undersea exploration and exploitation creates a demand for increasingly complex undersea data acquisition systems that must withstand deep ocean pressure. Two packaging approaches are possible.

1) The equipment can be sealed in rigid pressure-proof containers with pressure-proof seals.

2) The equipment can be constructed using pressuretolerant electronics (PTE) housed in a waterproof thin-walled container, oil-filled and pressure-balanced to the sea.

Fig. 1 [1] describes the pressures encountered in undersea applications. Since the pressure can be quite high, rigid pressure-proof containers are difficult to make, and a comparison of the two approaches show that packaging of PTE is a more cost-effective technique [2], [3]. Contributing to the advantages of packaging PTE are simplified sealing and near-zero pressure differential between the inside and outside.

With the advent of solid-state electronic equipment in the late 1950's, the possibility of operating electronics systems in an oil-pressure environment became apparent, and a number of feasibility programs were initiated by the Navy [4], [5] and

Manuscript received May 10, 1978; revised August 24, 1978.

The author is with the Heavy Military Electronics Department, General Electric Company, Syracuse, NY 13221.

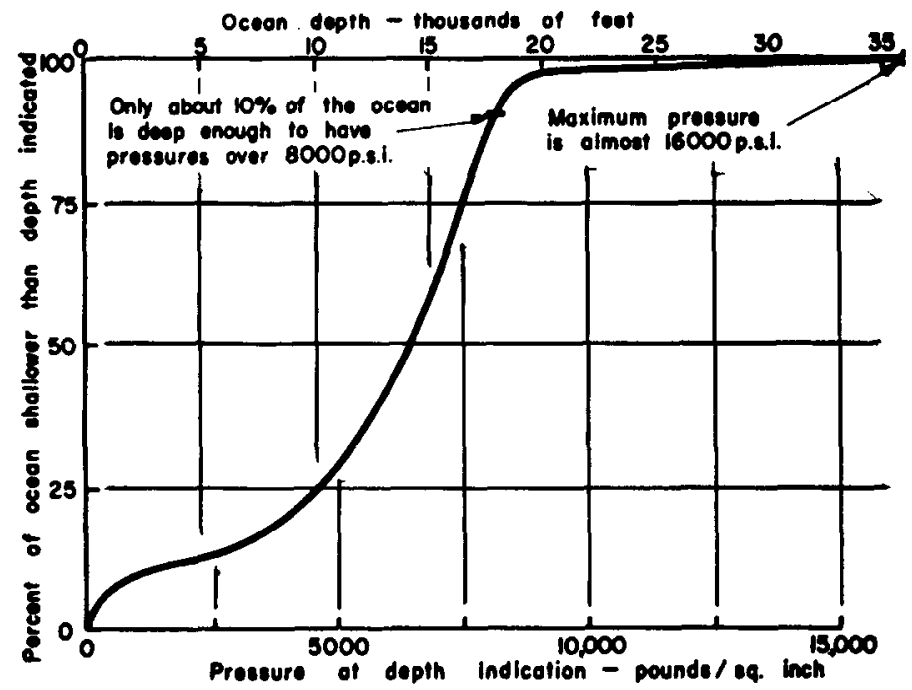

Fig. 1. Ocean bottom pressure.

industry [6] [8] . Investigations proved that the parameters of the transistor were essentially independent of pressure effects and inert oil had no serious short-term contaminating effect on the surface of passivated and barrier-coated semiconductors.

Extensive passive component testing followed, and a large number of components, virtually every class, were found to be useful at deep ocean pressure [9]. Generally speaking, components that are structurally homogeneous and free from air voids are potentially satisfactory PTE components. At the Navy Ship Research and Development Center at Annapolis, $\mathrm{MD}$, activity was focused on physical and physiochemical aspects of PTE [10]-[16]. The Naval Undersea Center at Hawaii performed electronic component device testing and characterization [17]-[22] .

Several manufacturers have applied PTE to relatively complex data acquisition systems. These helped establish PTE as a 